\title{
Diseño, desarrollo y evaluación preliminar de un novedoso monitor de signos vitales llevable para vacunos
}

\author{
Design, development and preliminary evaluation of a new wearable vital \\ signs monitor for cattle
}

\author{
Max Quispe Bonilla ${ }^{1}$, Adolfo Poma Gutiérrez ${ }^{2}$, Luis Serrano-Arriezu ${ }^{3}$, \\ Santiago Led Ramos ${ }^{3}$, Edgar Quispe Peña ${ }^{2,4}$
}

\section{Resumen}

\begin{abstract}
El monitoreo de los diferentes signos vitales en vacunos tiene importancia desde el punto de vista productivo, sanitario y de bienestar animal; sin embargo, existen pocos equipos que tengan el potencial de uso a nivel de campo y que no sean invasivos. Por tal motivo se llevó a cabo el presente trabajo con la finalidad de diseñar, construir y evaluar el uso de un pequeño MOnitor de SIgnos VItales LLevable (MOSIVILLe), que sea capaz de capturar las señales vitales en vacunos bajo condiciones de campo. El diseño y desarrollo del MOSIVILLe se realizó en Lima, Perú, entre enero y octubre de 2017 y la evaluación en campo se realizó en Chota, Perú, utilizando 11 vaquillas entre noviembre de 2017 y febrero de 2018. Con el uso del MOSIVILLe se obtuvieron las señales vitales de vacunos, reconociéndose las ondas $\mathrm{P}, \mathrm{Q}, \mathrm{R}, \mathrm{S}, \mathrm{T}$, complejo QRS e intervalo RR del electrocardiograma que condujeron a obtener la frecuencia cardiaca (FC). Adicionalmente, se obtuvo la temperatura de la piel $\left(\mathrm{T}^{\circ} \mathrm{P}\right)$ y la señal de ventilación con los picos de inhalación, exhalación, tiempo de inspiración y tiempo de espiración que determinan la frecuencia respiratoria (FR). El MOSIVILLe usado en vacunos en condiciones de campo permitió obtener una FC de $70.83 \pm 1.47$, FR de $25.24 \pm 1.64$ y una $\mathrm{T}^{\circ} \mathrm{P}$ de $31.52 \pm 0.40$ (promedio \pm error estándar), valores que se encuentran en concordancia con la literatura. Se concluye que el MOSIVILLe es una alternativa importante para obtener diversos signos vitales en vacunos bajo condiciones de campo.
\end{abstract}

Palabras clave: electrocardiograma; ECG; respiración; temperatura de la piel; equipo llevable

\footnotetext{
${ }^{1}$ Departamento Técnico, Maxcorp Technologies S.A.C., Lima, Perú

${ }^{2}$ Vicepresidencia de Investigación, Universidad Nacional Autónoma de Chota, Cajamarca, Perú

${ }^{3}$ Institute of Smart Cities, Departamento de Ingeniería Eléctrica, Electrónica y Comunicación, Universidad Pública de Navarra, Pamplona, España

${ }^{4}$ E-mail: edgarquispe62@gmail.com
}

Recibido: 19 de mayo de 2018

Aceptado para publicación: 25 de octubre de 2018 
The monitoring of various vital signs in cattle is important from the point of view of production, health and animal welfare; however, there are few devices that have the potential to be used at the field level and that are not invasive. For this reason, the present work was carried out with the purpose of designing, constructing and evaluating the use of wearable vital signs monitor (MOSIVILLe), which is capable of capturing the vital signs in cattle under field conditions. The design and development of MOSIVILLe was carried out in Lima, Peru, between January and October 2017 and the field evaluation was carried out in Chota, Peru, using 11 heifers between November 2017 and February 2018. The vital signs of cattle were obtained with the MOSIVILLe, recognizing the P, Q, R, $\mathrm{S}$, T waves, QRS complex and RR interval of the electrocardiogram that led to obtain the heart rate (HR). Additionally, the skin temperature $\left(\mathrm{T}^{\circ} \mathrm{S}\right)$ and the ventilation signal were obtained with the peaks of inhalation, exhalation, inspiration time and expiration time that determine the respiratory frequency (RF). MOSIVILLe used in cattle in field conditions allowed to obtain an HR of $70.83 \pm 1.47$, RF of $25.24 \pm 1.64$ and $\mathrm{T}^{\circ} \mathrm{S}$ of $31.52 \pm 0.40$ (mean \pm standard error), values that agree with the literature. It is concluded that MOSIVILLe is an important alternative to obtain various vital signs in cattle under field conditions.

Key words: electrocardiogram; ECG; breathing; skin temperature; wearable medical device

\section{INTRODUCCIÓN}

La monitorización de signos vitales resulta importante dentro de la producción y bienestar animal, pues mediante el conocimiento de los parámetros fisiológicos de personas y animales, tales como la frecuencia respiratoria, electrocardiograma, temperatura corporal, saturación de oxígeno, gasto cardiaco y presión arterial, entre otros, se pueden detectar problemas en la salud (Claxton, 1988; Husheer et al., 2016; Reddy y Sivajothi, 2016), así como evaluar la fisiología y el rendimiento productivo del animal (Radostits et al., 2009; Brugarolas et al., 2016), las cuales permiten tomar rápidas decisiones a fin de asegurar un bienestar favorable para todo ser vivo.

Existen numerosos tipos de tecnologías basadas en sensores que fueron desarrollados para el campo de la robótica, uso militar y defensa, así como para procesos industriales (Helwatkar et al., 2014), pero que han pasado a ser utilizadas en la monitorización de la salud que son usados en humanos (Niubó y Cruz, 2001) y animales (Helwatkar et al., 2014). Es así que se ha pasado del uso de una serie de dispositivos complejos, voluminosos y costosos, que pueden medir varios parámetros fisiológicos con precisión a tener diversos monitores de signos vitales portables y llevables, cada uno con su peculiaridad, para mascotas (Menkes, 2013), vacunos (Hixson, 2007; Husheer et al., 2016) y caballos (Khelifi et al., 2017; Paulussen et al., 2017), con tendencia casi general para apoyar la producción intensiva y automatizada, así como el bienestar animal.

En el caso de los vacunos se ha logrado captar y monitorizar diversos signos vitales. Los podómetros son sensores simples y baratos que permiten detectar el estado de la actividad de una vaca, siendo utilizados incluso para identificar el comportamiento estral (Lovendahl y Chagunda, 2010). Los sensores de posicionamiento GPS también se emplean para identificar estados de movimiento en la vaca, tales como el caminar, comer, echarse y descansar, entre otros (Godsk y Kjærgaard, 
2011). Asimismo, los acelerómetros son actualmente los candidatos más prometedores para proveer datos precisos del movimiento de las vacas al pastoreo, el cual fue propuesto tiempo atrás por Bando (1997).

De otro lado, para determinar signos vitales como la frecuencia y el pulso cardiaco, se realizan mediciones de la actividad eléctrica del corazón o de potencial eléctrico entre dos puntos del cuerpo, captados mediante un electrocardiógrafo, que ofrece una medición precisa de la frecuencia cardiaca, así como una información detallada acerca de la actividad de los músculos del corazón. De este modo, dos o más electrodos pueden ser adheridos al pecho mediante unas correas que captan las señales eléctricas del corazón y luego son amplificadas y procesadas para su visualización en una pantalla LCD de una computadora, reloj o celular (Pallas et al., 2010; Husheer et al., 2016).

La frecuencia respiratoria puede ser medida de forma indirecta, a través de la saturación de oxígeno en la sangre, o en forma directa a través de neumotacógrafos que pueden medir la expansión y contracción del pecho o que miden los cambios de resistencia eléctrica a través de la cavidad del pecho. Otros tipos de aparatos miden directamente la inhalación y exhalación a través de la boca del paciente o de un tubo de respiración (Husheer et al., 2016). Adicionalmente, existen alternativas como la espirometría por sensores de desplazamiento y la neurografía por impedancia. Esta última provee información cualitativa de los movimientos torácicos (Olmos et al., 2007). La impedancia neumográfica emplea bajas amplitudes y altas frecuencias $(50$ a $500 \mathrm{kHz})$ de corriente alterna entre dos superficies de electrodos para registrar los movimientos torácicos o cambios de volumen en la caja torácica durante un ciclo respiratorio, basado en la Ley de Ohm, en la cual la caída de voltaje a través de los electrodos es imputada como impedancia, el cual incrementa durante la inspiración y disminuye durante la espiración (Landon, 2003).
La forma de las ondas del electrocardiograma variará dependiendo de las derivaciones consideradas en función a la ubicación de los electrodos. Es así que DeRoth (1980) trabajando con 12 derivaciones logró obtener alrededor de siete ondas $\mathrm{P}, \mathrm{T}$ y complejo QRS en vacas al colocar los electrodos negativo y positivo en el ángulo caudal de la escápula derecha y el $5^{\circ}$ espacio intercostal a nivel de la unión costocondral izquierdo, respectivamente, conocida como derivación ápice-base. Bajo esta consideración, Reddy y Sivajothi (2016) caracterizaron el electrocardiograma de vacunos lecheros, encontrando siempre positivo la onda $\mathrm{P}$, mientras que la amplitud de la onda R siempre fue negativa. Bajo ese mismo criterio, otros investigadores determinaron la frecuencia cardiaca en función de la distancia de los picos R-R, habiendo aparecido en la medicina veterinaria varias publicaciones sobre parámetros de ECG y ritmo cardiaco de varias razas de vacunos de carne y de leche (Mendes et al., 2001; Rezakhani et al., 2004; Reddy y Sivajothi, 2016).

En este contexto, el desarrollo de los sensores y el procesamiento de datos han dado lugar a una serie de monitores de signos vitales que tienen gran utilidad en la gestión y producción de ganado vacuno, teniendo cada uno sus peculiaridades, pues unos determinan movimientos (Lovendahl y Chagunda, 2010; Godsk y Kjærgaard, 2011), otros permiten la visualización del electrocardiograma bajo diferentes tipos de electrodos (Albulul, 2016), otros determinan gasto cardiaco, frecuencia respiratoria y saturación de oxígeno (DeRoth, 1980; Mendes et al., 2001, Rezakhani et al., 2004; Reddy y Sivajothi, 2016). Sin embargo, se requieren de equipos que permitan monitorizar varios signos vitales sin perturbar al animal y que a la vez sea llevable. Por este motivo, el presente trabajo tuvo como finalidad diseñar, desarrollar y evaluar preliminarmente un monitor de signos vitales (MOSIVILLe) para su uso en vacunos, con utilidad práctica en la salud, producción y bienestar animal. 


\section{Materiales y Métodos}

\section{De la Ubicación y Tiempo}

El diseño y desarrollo del MOSIVILLe (MOnitor de SIgnos VItales LLevable) se realizó en el Laboratorio de Maxcorp Technologies S.A.C, ubicado en la ciudad de Lima, Perú, entre enero y octubre de 2017. La evaluación en campo se realizó en el establo bovino de Colpahuacaríz, de la Universidad Nacional Autónoma de Chota, Perú. Se utilizaron 11 vaquillas ( 5 Holstein y 6 criollas), con edades entre 14 y 20 meses, a los que se colocó MOSIVILLe (Figura 1) en febrero de 2018.

\section{Del Diseño para la Construcción}

Para el diseño del MOSIVILLe se consideraron: a) Una tarjeta electrónica de cuatro capas, que permita adquirir las señales cardiacas, ventilatorias y de temperatura, que luego de procesarlas y almacenarlas pueda enviar los datos obtenidos a una computadora mediante protocolos de comunicación USB; b) Una parte mecánica, cuya finalidad fue proteger la tarjeta electrónica y permitir una fácil adhesión del MOSIVILLe a una correa elástica, que pueda ser colocada alrededor del tórax del animal (Figura 2) a fin de capturar las señales de los signos vitales, y c) una interfaz gráfica del usuario (GUI) para la visualización y evaluación de las señales obtenidas (Figura 3).

\section{Del Componente Electrónico}

Para la construcción de la tarjeta electrónica se utilizaron dispositivos electrónicos como un integrado ADS1292R, un microcontrolador ATSAMD21G18A-AU, dispositivos electrónicos de montaje superficial, sensor de temperatura, tarjeta microSD, resistencias, capacitores e inductores. También se implementaron los protocolos de comunicación USB y de almacenamiento. Toda la programación del firmware se realizó en el programa Atmel Studio 6. Los dispositivos electrónicos SMD (dispositivos de montaje superficial) se soldaron a la tarjeta electrónica, según el diseño electrónico elaborado en el programa Altium Designer (Figura 4). El sensor se soldó a la tarjeta electrónica del ECG de tal manera que, al colocar el dispositivo en el tórax del animal, la luz infrarroja emitida tenga contacto directo con la superficie de la piel.

\section{Del Componente Mecánico}

Mediante el programa Autodesk Inventor se diseñó una carcasa que permite la protección de la tarjeta electrónica y la fácil adhesión al tórax del animal. Esta carcasa se imprimió con una impresora 3D en material PLA (Figura 3). Para la adhesión de MOSIVILLe al animal se usó una correa elástica, a la cual se le añadió tres broches para colocar los electrodos secos o de gel (que funcionan como polos eléctricos) y otros tres broches para acoplarlo con el MOSIVILLe. Los broches se conectaron en pares al interior de la correa, de tal manera que cada broche de la tarjeta electrónica coincida con un electrodo seco o de gel.

\section{Del Componente Informático}

La interfaz gráfica del usuario (Graphical User Interface, GUI), se desarrolló en lenguaje C Sharp. La GUI tuvo como finalidad la visualización y evaluación de las señales cardiacas, ventilatorias y de temperatura. También se implementaron botones y ventanas para la configuración de alarmas (bradicardia, taquicardia, hipoventilación, hiperventilación, fiebre), carga de archivos, filtros, zoom, y desplazamiento, entre otras opciones. A este software propietario se le denominó SECG (Software del EleCtrocardióGrafo).

\section{Uso del MOSIVILLe}

Las vaquillas fueron rasuradas a nivel de cada broche de la correa donde se colocan los electrodos secos o de gel (aproximadamente $6 \mathrm{~cm}$ de diámetro), a fin de captar 


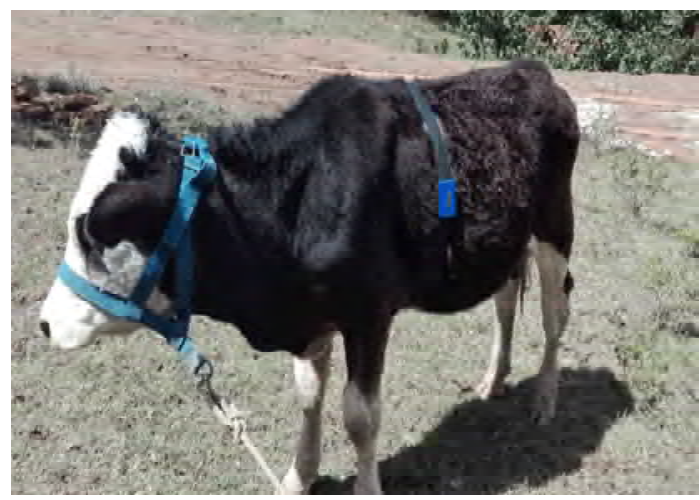

Figura 1. MOSIVILLe colocado en una vaquilla criolla mediante una correa elástica. Véase el tamaño pequeño del dispositivo

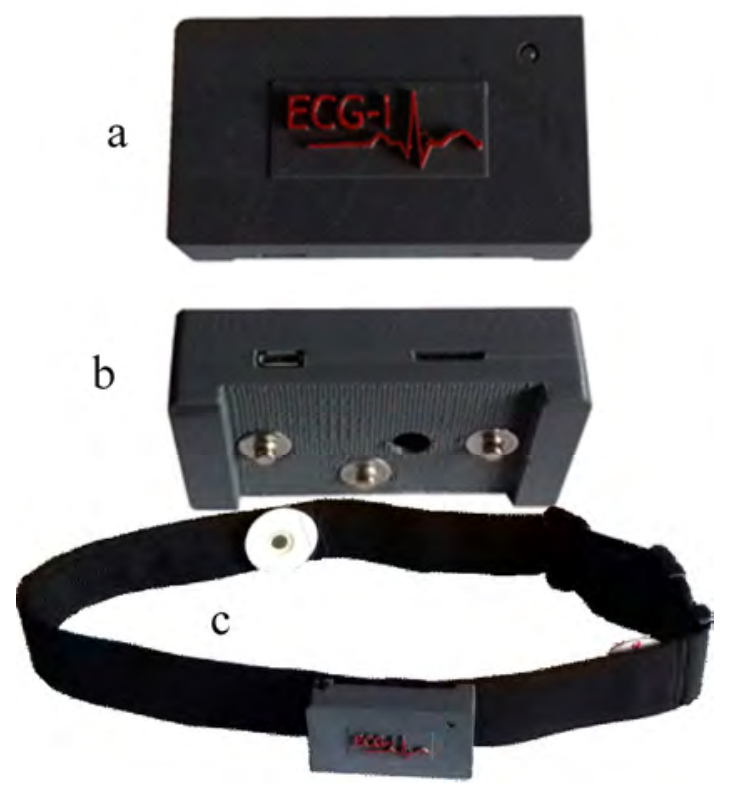

Figura 2. Monitor de signos vitales llevable MOSIVILLe. a: Lado frontal; b: Lado posterior; c: Correa donde va adherida el dispositivo

señales limpias o con pocos artefactos. Las zonas para rasurar fueron en el séptimo espacio intercostal a nivel de la unión costocondral, otra en la unión costo vertebral, ambas en el flanco izquierdo, y la última en el séptimo espacio intercostal a nivel de la última unión costo vertebral del flanco derecho. Este último punto actúa como electrodo de referencia para disminuir el nivel de interferencia o ruidos inherentes al propio animal.
Se colocó la correa elástica alrededor del tórax de cada animal, teniendo en cuenta una tensión que no incomode al animal pero que fije adecuadamente los electrodos. Luego se colocó el MOSIVILLe mediante los broches de sujeción (Figura 2). Para el inicio de la captura de información se presiona el pulsador de MOSIVILLe que, luego de aproximadamente 30 segundos, inicia el almacenamiento de las señales cardiacas, 


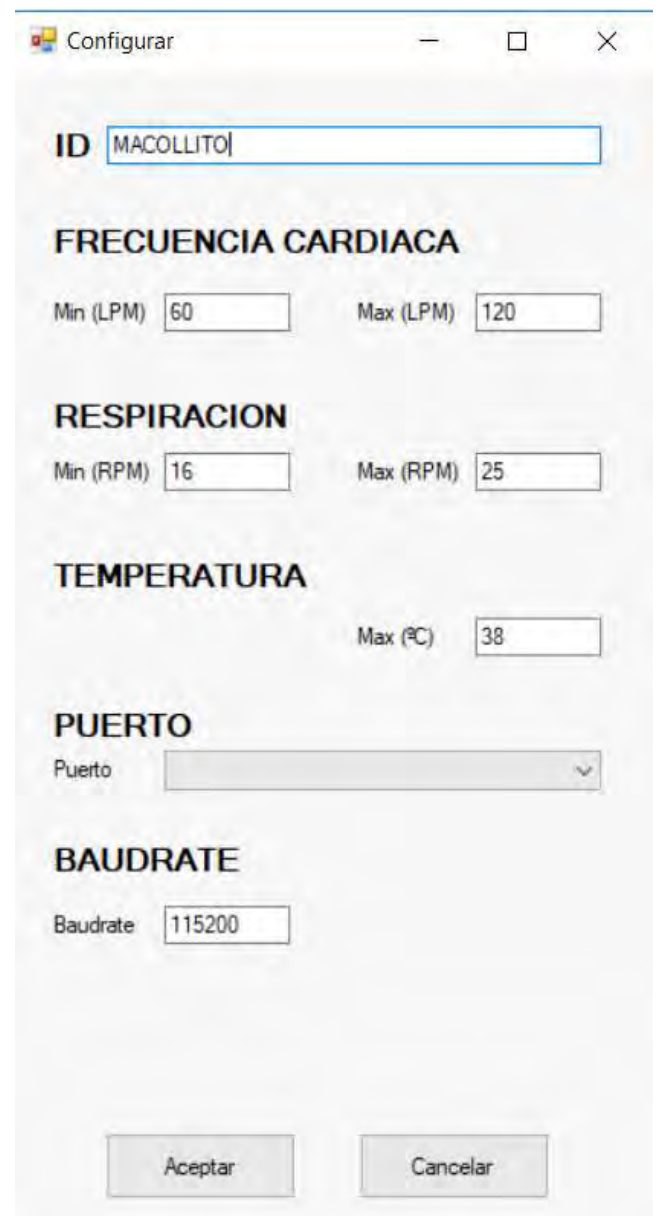

Figura 3. Interfaz gráfica del usuario de la opción CONFIGURAR en el software SECG

ventilatorias y de temperatura. Para finalizar el monitoreo se presiona nuevamente el pulsador.

Para identificar las señales obtenidas de cada animal, previo al inicio de captura de las señales, se ingresó mediante un clic en la opción de configurar en el software SECG, una nueva interfaz de usuario (Figura 5), en donde se anota la identificación (ID) del animal a evaluar o monitorear.

Para visualizar las señales vitales obtenidas, se conectó el MOSIVILLe, mediante un cable USB, a la computadora que tenía instalado el Software SECG de visualización. Luego, al pulsar en el botón «Explorar» del
SECG se ubica el archivo donde se almacenaron los datos e inmediatamente se visualizan las señales vitales de cada animal (Figura 6).

\section{Pruebas de MOSIVILLe}

Para la evaluación y uso del MOSIVILLe se utilizaron las 11 vaquillas, bajo condiciones entre 12 y $24^{\circ} \mathrm{C}$ (media: $18.5^{\circ} \mathrm{C}$ ) de temperatura ambiental y entre 54 a $72 \%$ (media: $61.3 \%$ ) de humedad relativa ambiental. Estos registros se obtuvieron de una estación meteorológica portátil de ultra-precisión (Oregon Scientific, modelo WMR300). Asimismo, se utilizó un termómetro de bulbo de mercurio para determinar la 


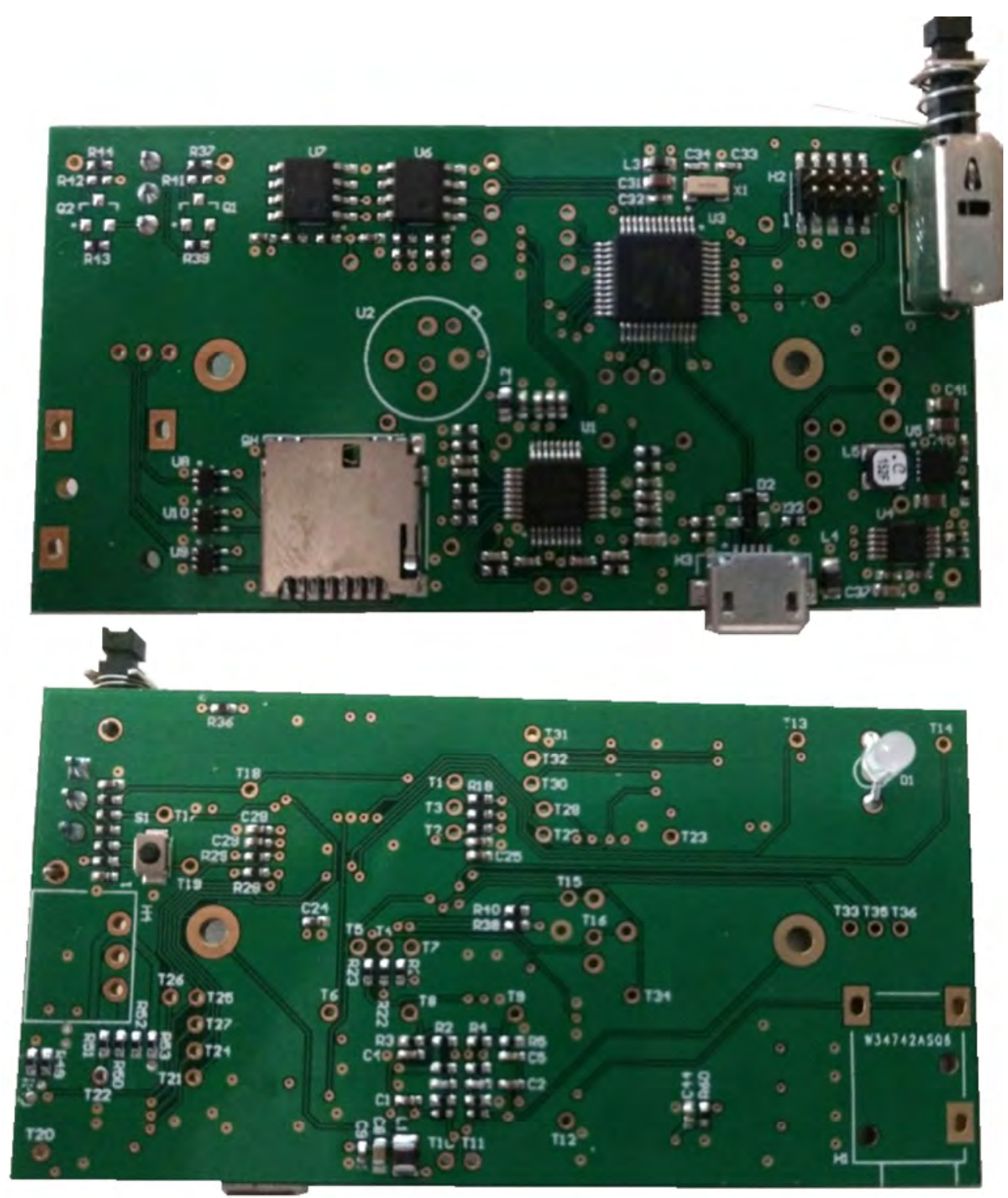

Figura 4. Capa superior e inferior (arriba y abajo, respectivamente) de la tarjeta electrónica del MOSIVILLe

temperatura rectal del animal, el cual fue registrado durante el monitoreo, lo cual sirvió para comparar con la temperatura de la piel obtenida con el MOSIVILLe.

\section{Análisis Estadístico}

Se utilizo el software libre R v. 3.1.1 (R Core Team, 2016) para la estadística descriptiva de la frecuencia cardiaca, frecuencia respi- ratoria, temperatura de la piel y temperatura rectal. Los datos fueron posteriormente comparados con lo indicado en la bibliografía.

\section{Resultados}

La Figura 3 muestra el MOSIVILLe desarrollado, el cual tiene un peso de $55.75 \mathrm{~g}$ y mide $7.8 \mathrm{~cm}$ de largo, $3.9 \mathrm{~cm}$ de ancho y 


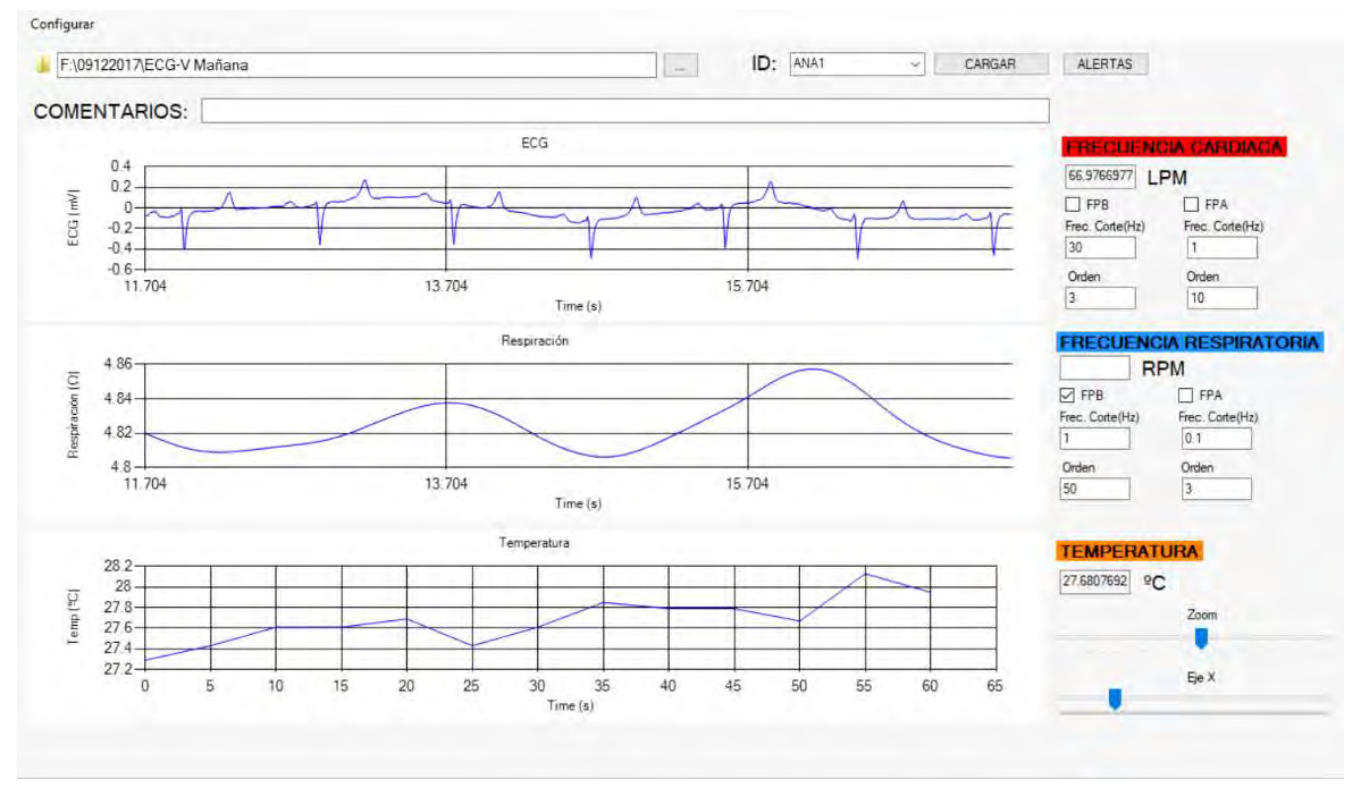

Figura 5. Interfaz gráfica del usuario del MOSIVILLe donde se observan las señales vitales captadas en vacunos. Parte superior: electrocardiograma. Parte media: señales de respiración. Parte inferior: la fluctuación del monitoreo de la temperatura de la piel. En la parte derecha se observan los promedios de los valores de las señales vitales en monitoreo

$1.9 \mathrm{~cm}$ de grosor. Al estar adherido a una correa permite ser llevado por el animal mientras se encuentre realizando sus actividades rutinarias, razón por la cual puede ser considerado un aparato llevable. Asimismo, los electrodos positivo y negativo en la correa y adosados a nivel del $7^{\circ}$ espacio intercostal en la unión costo condral y en la unión costo vertebral respectivamente, permiten obtener una derivada base-ápice modificada (B1-A), que resulta similar a la base-ápex determinada por DeRoth (1980).

El núcleo central del MOSIVILLe es una tarjeta electrónica de cuatro capas (Figura 4) el cual consta de: un integrado ADS1292R (3.7Vdc) multicanal, de muestreo simultáneo con convertidor analógico/digital (ADC) sigma delta $(\Delta \Sigma)$ de 24-bit, con amplificador de ganancia programable (PGA), referencia interna, y un oscilador de $512 \mathrm{kHz}$ que permite registrar las señales cardiacas y de ventilación; un microcontrolador ATSAMD21G18A-AU de baja potencia $(1.62 \mathrm{Vdc}-3.63 \mathrm{Vdc})$ con un procesador ARM Cortex-M0+ de 32 bits, con una memoria Flash de $256 \mathrm{kB}$ y $32 \mathrm{kB}$ de memoria SRAM, que actúa como el núcleo de inteligencia, la que tiene como finalidad configurar y procesar la data en bruto obtenida del integrado ADS1292R, implementado con algoritmos para detectar picos de las señales, la frecuencia cardiaca, curvas de espiración e inspiración, filtros pasa bajo, pasa alto y pasa ban$\mathrm{da}$, alarmas de extremos (para indicar bradicardias o taquicardias); un cargador de batería BQ24090DGQR que permite cargar la batería LiPo recargable (3.7V, $1000 \mathrm{mAh})$ y a su vez es la fuente de energía para toda la tarjeta electrónica; una tarjeta microSD de 4B, fácilmente intercambiable para el almacenamiento de señales; un sensor de temperatura infrarrojo MLX90614ESF con una resolución de $0.02{ }^{\circ} \mathrm{C}$ y rango entre $-70 \mathrm{y}$ 


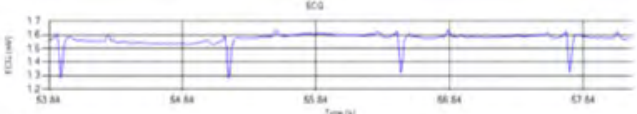

Kiara

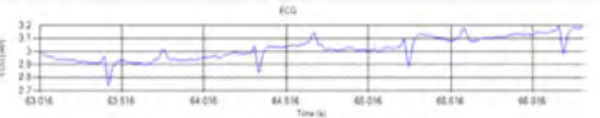

Kina

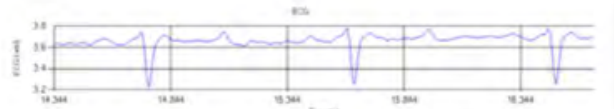

Ans

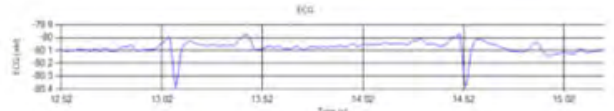

Nilda

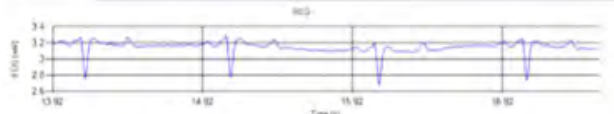

Zulma

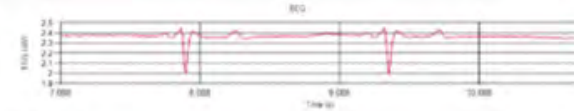

Juana

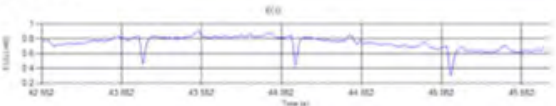

Linda

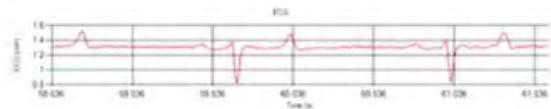

Tabita

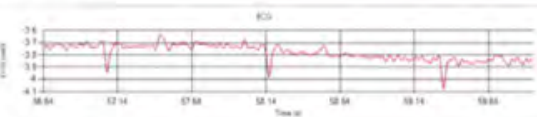

Magal

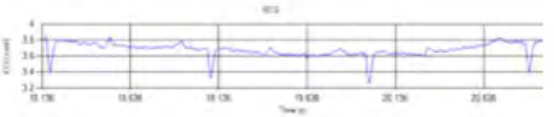

Laura

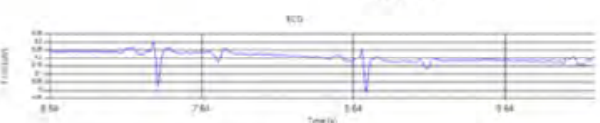

Iris

Figura 6. Electrocardiogramas obtenidos con el MOSIVILLe en vaquillas Holstein y criollas. Obsérvese la característica del complejo QRS que en todas tiene una configuración negativa. En 10 de las 11 vaquillas monitoreadas la onda T fue positiva

Cuadro 1. Estadísticos de constantes fisiológicas de vacunos evaluadas en condiciones de campo, obtenidas con MOSIVILLe ( $\mathrm{n}=11)$. Además, se presenta la temperatura rectal obtenida con un termómetro rectal

\begin{tabular}{lccccc}
\hline & $\begin{array}{c}\text { Frecuencia } \\
\text { cardiaca }\end{array}$ & $\begin{array}{c}\text { Frecuencia } \\
\text { respiratoria }\end{array}$ & $\begin{array}{c}\text { Temperatura } \\
\text { rectal }\end{array}$ & $\begin{array}{c}\text { Temperatura } \\
\text { de piel }\end{array}$ & $\begin{array}{c}\text { Diferencia } \\
\text { en temp. }\end{array}$ \\
\hline Promedio & 70.83 & 25.24 & 38.81 & 31.52 & 7.29 \\
D.E. & 8.30 & 9.27 & 0.39 & 2.27 & 2.20 \\
Mínimo & 48.0 & 6.70 & 38.0 & 27.30 & 3.06 \\
Máximo & 90.7 & 50.00 & 39.50 & 35.30 & 11.30 \\
Rango & 42.7 & 43.30 & 1.50 & 8.00 & 7.70 \\
\hline
\end{tabular}

$+380{ }^{\circ} \mathrm{C}$, que permite captar la temperatura superficial; un diodo LED bicolor R/G como indicador de encendido/apagado, alarmas y detección de señal. La tarjeta también contiene otros dispositivos electrónicos como resistencias, capacitores e inductores.
El MOSIVILLe tiene la capacidad de registrar las distintas ondas, segmentos e intervalos del electrocardiograma (Figura 5) y en torno a ella realiza el contaje automático de los intervalos R-R que los traduce en función a un minuto como la frecuencia cardiaca. 
Asimismo, basado en la impedancia pletismográfica utilizando una corriente de excitación de $30 \mathrm{uA}$ en una frecuencia de 32 $\mathrm{kHz}$ permite registrar las ondas de inspiración y espiración durante un periodo de tiempo establecido. Adicionalmente se registran los cambios de temperatura de la piel del animal, obteniendo un promedio durante el tiempo que duró el monitoreo.

El aparato también permite configurar la frecuencia cardiaca mínima y máxima para identificar bradicardias y taquicardias; frecuencia respiratoria mínima y máxima para identificar hipoventilación e hiperventilación, y temperatura máxima o mínima para identificar fiebre o hipotermia. La visualización de estas patologías, de haberlas, se muestra en color rojo en cada señal obtenida, para su rápida identificación.

Las amplitudes y duración de las despolarizaciones y repolarizaciones de las diferentes partes del tejido muscular cardiaco que conducen a la elaboración del electrocardiograma son registrados en el MOSIVILLe como datos en una hoja Excel en forma automática, de modo que es posible realizar diversas evaluaciones del electrocardiograma, tales como la medición de las amplitudes de las ondas y duración de los periodos, intervalos y segmentos.

Los electrocardiogramas obtenidos con el MOSIVILLe en las 11 vaquillas presentaban una onda $\mathrm{P}$ positiva, no observándose la onda $\mathrm{Q}$, pero si una onda $\mathrm{R}$ de amplitud similar o menor a la onda $\mathrm{P}$, resaltando una mayor amplitud la onda $\mathrm{S}$, razón por la cual el complejo QRS resulta tener una forma negativa. Solo se encontró un solo caso de onda T negativa, pero que, en todos los casos, dicha onda tuvo una mayor amplitud que la onda $\mathrm{P}$ (Figura 6).

Los resultados del monitoreo de los signos vitales obtenidos con el MOSIVILLe se muestran en el Cuadro 1. Se aprecian los promedios de 70.83 latidos/min (lpm), 25.24 respiraciones/min (rpm) y $31.52{ }^{\circ} \mathrm{C}$, respectiva- mente para la frecuencia cardiaca, frecuencia respiratoria y temperatura de piel. Asimismo, se aprecia que la temperatura rectal resulta ser menos variable que la temperatura de piel, existiendo en torno a ella una diferencia promedio de $7.29^{\circ} \mathrm{C}$.

\section{Discusión}

Existen otros monitores de signos vitales en vacunos (Hixson, 2007; Paulussen et al., 2017); sin embargo, el MOSIVILLe tiene la particularidad de ser llevable, debido a sus dimensiones y peso, y permite capturar señales electrocardiográficas, frecuencia cardiaca, frecuencia respiratoria y temperatura de piel en forma simultánea, lo cual le confiere la capacidad de ser utilizado para detectar arritmias cardiacas (Rezakhani et al., 2004), diversas enfermedades del corazón (Mohapatra et al., 2017), estado de actividad del animal (Bewley et al., 2010), disturbios electrolíticos y modificaciones del volumen cardiaco (DeRoth, 1980), así como temperatura corporal (Hixson, 2007), entre otros estados.

Aunque trabajos anteriores indican que los ECG registrados usando derivaciones de las extremidades anteriores en ganado tienen bajas polaridades y gran variabilidad (Rezakhani et al., 2004), lo cual podría deberse a la posición de los electrodos en relación a la posición del corazón en la cavidad torácica (DeRoth, 1980) y a la distribución del sistema de conducción en el miocardio (Abramson y Margolin, 1936), que causaría la cancelación de algunas ondas; sin embargo, los electrocardiogramas captados con el MOSIVILLe resultan tener baja variabilidad. Es así que más del $90 \%$ de las vaquillas del estudio tuvieron las mismas características respecto a la visualización de las ondas $\mathrm{P}, \mathrm{T}$ y complejo QRS, comparado con otras derivaciones reportadas por DeRoth (1980) (Figura 7). Estas características resultan similares con lo encontrado en vacas Holstein por otros autores (DeRoth, 1980; Rezakhani et al., 

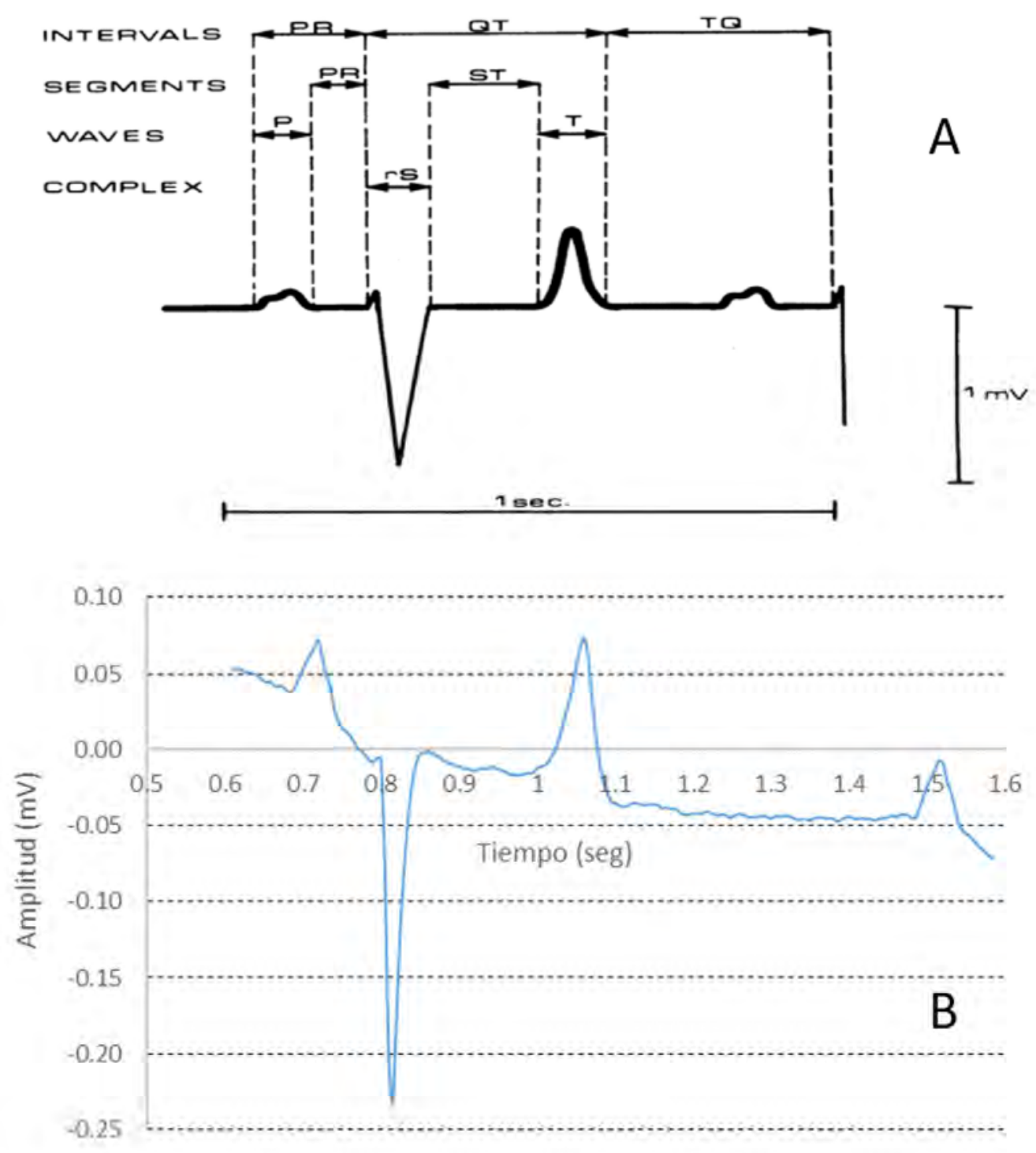

Figura 7. Electrocardiogramas de vacuno. A: Electrocardiograma correspondiente a la derivada base-ápice obtenida por DeRoth (1980), donde se indican los intervalos, segmentos, ondas y complejos. B: Electrocardiograma obtenido con MOSIVILLe de la derivada base-ápice modificada en una de las vaquillas en estudio, de la base de datos de Excel. Véase la similitud entre A y B

2004; Reddy y Sivajothi, 2016) quienes utilizaron la derivada base-ápice. También es similar a lo reportado en terneros Holstein por Mendes et al. (2001), quienes obtuvieron el complejo QRS negativo, con Q negativo, R positivo y con amplitud $S$ negativa casi el doble de Q y R, razón por la cual tiene una configuración negativa, y P positivo de baja amplitud. Asimismo, también es similar a lo reportado por Mohapatra et al. (2017), quienes también presentan un complejo QRS negativo en vacas Jersey, e incluso a lo reportado en caballos de paso por Mira et al. (2016), quienes también se basaron en la derivada base-ápice. 
De otro lado, los resultados de frecuencia cardiaca son ligeramente menores a los parámetros reportados por Rezakhani et al. (2004), quienes obtienen un promedio de $75.73 \pm 9.13 \mathrm{lpm}$, aunque son bastante próximos con el resultado de Reddy y Sivajothi (2016), quienes reportaron una frecuencia cardiaca de $71.65 \mathrm{lpm}$ en vacunos entre 2 y 4 años. Estas pequeñas variaciones podrían deberse al tipo de electrodo utilizado, ya que es conocido que los electrodos tipo pinza de «lagarto» ocasionan cierta incomodad al animal, que podría verse reflejado en un ligero incremento de la frecuencia cardiaca.

Con relación a la frecuencia respiratoria, los valores del estudio se encuentran entre 24 y $36 \mathrm{rpm}$, que es el reportado para bovinos normales (Ferreira et al., 2006), y concordante con Nascimento et al. (2013), quienes reportan frecuencias entre 12 y $36 \mathrm{rpm}$. Sobre la temperatura corporal, los resultados encontrados son similares a lo encontrado por Cruz et al. (2016), quienes reportan valores de temperatura de piel entre 29 y $31{ }^{\circ} \mathrm{C}$ con diferencias respecto a la temperatura rectal alrededor de $8{ }^{\circ} \mathrm{C}$, mientras que Martello et al. (2004) reportaron temperaturas de piel entre 31 y $32{ }^{\circ} \mathrm{C}$ durante la mañana en vacunos Holstein; comparaciones que conlleva a indicar que el monitoreo realizado con el MOSIVILLe es adecuado.

Aunque se ha demostrado que el MOSIVILLe capta bien las señales electrocardiográficas, es conveniente realizar algunas mejoras como una activación de su funcionamiento a distancia, pues los animales se incomodan con la cercanía de las personas, más aún cuando son animales criados bajo condiciones extensivas. Asimismo, es recomendable que la lectura de los signos vitales sea en tiempo real, por la cual debería implementarse al MOSIVILLe con tecnologías de comunicación inalámbrica como WiFi o bluetooth, permitiendo así la configuración y registro de señales de manera inalámbrica desde un celular inteligente (Smartphone), tableta o computadora.

\section{Conclusiones}

- El MOSIVILLe es un equipo que permite registrar las diferencias de potencial que ocurren sobre la superficie del cuerpo del animal como resultado de la actividad eléctrica de los músculos del corazón, que luego son procesados para obtener un electrocardiograma en bovinos.

- La derivada base-ápice modificada permite obtener ECG homogéneos en vacunos, con ondas $\mathrm{P}$ y $\mathrm{T}$ siempre positivos y una onda $\mathrm{S}$ bastante pronunciada pero negativa.

- El MOSIVILLe presenta valores de frecuencia cardiaca, frecuencia respiratoria y temperatura corporal similares a los indicados en la literatura científica.

- Se puede afirmar que el MOSIVILLe se convierte en una buena alternativa para uso dentro de la clinica veterinaria, así como para estudios practicos y de investigación de bienestar y producción animal.

\section{Agradecimientos}

Los autores manifiestan su agradecimiento a CIENCIACTIVA de CONCYTEC por el apoyo financiero a través del Contrato de Cofinanciamiento N. ${ }^{\circ}$ 159-2016FONDECYT. Así mismo, a René Hinojosa por su apoyo en el trabajo de campo.

\section{Literatura Citada}

1. Abramson DI, Margolin S. 1936. A Purkinje conduction network in the myocardium of the mammalian ventricles. J Anat 70: 250-259.

2. Albulul A. 2016. Evaluating major electrode types for idle biological signal measurements for modern medical technology. Bioengineering 3: 20. doi: 10.3390/bioengineering 3030020 
3. Bando T. 1997. Grazing stock managing apparatus and grazing stock managing system using the apparatus. Patente JPH1156146A. Japón.

4. Bewley JM, Boyce RE, Hockin J, Munksgaard L, Eicher SD, Einstein ME, Schutz MM. 2010. Influence of milk yield, stage of lactation, and body condition on dairy cattle lying behaviour measured using an automated activity monitoring sensor. J Dairy Res 77: 1-6. doi: 10.1017/S0022029909990227

5. Brugarolas R, Latif T, Dieffenderfer J, Walker K, Yuschak S, Sherman BL, Roberts DL, et al. 2016. Wearable hear rate sensor systems for wireless canine health monitoring. IEEE Sens J 16:34553464. doi: 10.1109/JSEN.2015.2485210

6. Claxton MS. 1988. Electrocardiographic evaluation of arrhythmias in six cattle. J Am Vet Med Assoc 192: 516-521.

7. Cruz PFF, Monteiro CP, Guimaraes EC, Antunes RC, do Nascimento MRB. 2016. Physiological parameters, hair coast morphological characteristics and temperature gradients in Holstein-Gyr crossbred cows. Biosci J 32: 471-477.

8. DeRoth L. 1980. Electrocardiographic parameters in the normal lactating Holstein Cow. Canadian Vet J 21: 271-277.

9. Ferreira F, PiresII MFA, Martinez $M L$, Coelho SG, Carvalho $A U$, Ferreira PM, Facury Filho EJ, Campos WE. 2006. Parâmetros fisiológicos de bovinos cruzados submetidos ao estresse calórico. Arq Bras Med Vet Zootec 58: 732-738. doi: 10.1590/S010209352006000500005

10. Godsk T, Kjaergaard MB. 2011. High classification rates for continuous cow activity recognition using low-cost GPS positioning sensors and standard machine learning techniques. $11^{\text {th }}$ Industrial Conferencia, ICDM. New York, USA.

11. Helwatkar A, Riordan D, Walsh J. 2014. Sensor technology for animal health monitoring. Proc $8^{\text {th }}$ International Conference on Sensing Technology. Liverpool, UK.
12. Hixson T. 2007. Vital signs monitoring system for animals. Patente 0159342A1. USA.

13. Husheer SLG, Hayes JM, Chausiaux OE. 2016. Monitoring vital signs. PCT/ GB2016(050591. Cambridge. Gran Bretaña.

14. Khelifi A, Hamli RA, Tamimi SA, Ali RA. 2017. An automated system for monitoring horses vital signs using heart beat sensors. 2017 Palestinian International Conference on Information and Communication Technology (PICICT). Gaza City, Palestinian Authority.

15. Landon C. 2003. Respiratory monitoring: advantages of inductive plethysmo-graphy over impedance pneumography. VivoMetrics. $7 \mathrm{p}$.

16. Lovendahl P, Chagunda MG. 2010. On the use of physical activity monitoring for estrus detection in dairy cows. J Dairy Sci 93:249-259. doi: 10.3168/jds.2008-1721

17. Martello L, Savastano J, Silva S, Titto EA. 2004. Respostas fisiológicas e produtivas de vacas holandesas em lactação submetidas a diferentes ambientes. R Bras Zootec 33: 181-191. doi: 10.1590/S1516-35982004000100022

18. Mendes LCN, Camacho AA, Alves ALG, Borges AS, Souza RCA, Ferreira WL. 2001. Standard electrocardiographic values in Holstein calves. Arq Bras Med Vet Zoo 53: 641-644. doi: 10.1590/S0102-09352001000600002

19. Menkes A. 2013. Pet animal collar for health \& vital signs monitoring, alert and diagnosis. Patent 001470.6A1. USA.

20. Mira J, Posada S, Castillo CA, Saldarriaga A. 2016. Parámetros electrocardiógráficos en caballos de raza criollo colombiano, pacientes de un establecimiento de Antioquia, Colombia. Rev Med Vet 32: 39-51. doi: 10.19052/mv.3854

21. Mohapatra S, Jyotiranjan T, Khadanga AP, Kundu AK. 2017. A comparative evaluation of the base apex lead electrocardiogram in young and adult crossbred cows of Odisha. Pharm Innovat J 6: 113-115. 
22. Niubó I, Cruz A. 2001. Evaluación de un monitor de paciente. Rev Cubana Invest Biomed 20: 128-135.

23. Nascimento GV, Cardoso EA, Batista N, Souza BB, Cambui GB. 2013. Indicadores productivos, fisiológicos e comportamentais de vacas de leite. Agropec Cient Semiarido 9: 28-36.

24. Olmos D, Rodríguez JL, Gaitán MJ, Pimentel AB. 2007. Neumografia por impedancia como alternativa para la valoración de la apnea obstructiva del sueño. Rev Mex Ing Biomed 28: 36-43.

25. Pallas AR, Piedrafita JO, Cassanella AR, Gomez CJ, Díaz CD. 2011. Método y aparato para obtener el electrocardiograma mediante electrodos secos dobles. Patente WO2011104398A1. Universidad Politécnica de Cataluña. España.

26. Paulussen E, Coppola G, Marie de Lang P, Schipper A. 2017. Animal vi- tal signs monitor. Patente US 2017/ 0127959A1. Eindhoven, Netherlands.

27. $R$ Core Team. 2017. R: a language and environment for statistical computing. $\mathrm{R}$ Foundation for Statistical Computing, Vienna, Austria. [Internet]. Available in: https://www.R-project.org/

28. Radostits OM, Gay CC, Hinchcliff $K W$, Constable PD. 2009. Veterinary medicine: a text book of the diseases of cattle, horses, sheep, pigs and goats. $10^{\text {th }}$ ed. USA: WB Saunders. 2065 p.

29. Reddy BS, Sivajothi S. 2016. Electrocardiographic parameters of nor mal dairy cows during diferente ages. $\mathrm{J}$ Vet Sci Med 4: 1-5.

30. Rezakhani A, Paphan AA, Shekarfroush S. 2004. Analysis of base apex lead electrocardiograms of a normal dairy cows. Vet Arhiv 74: 351-358. 